\title{
Temporal evolution of different temperature plasma during explosive events
}

\author{
M. S. Madjarska and J. G. Doyle
}

\author{
Armagh Observatory, College Hill, Armagh BT61 9DG, N. Ireland
}

Received 18 July 2001 / Accepted 9 November 2001

\begin{abstract}
High cadence observations (10 s exposure time) obtained with the SUMER spectrometer on-board SoHO in the Ly 6 (20 $000 \mathrm{~K})$ and S VI $(200000 \mathrm{~K})$ lines reveal new insight on the nature of explosive events. A time delay in the response of the S vi line with respect to the Ly 6 line has been observed, with the Ly 6 line responding with about 20-40 s earlier. A temporal series obtained with $30 \mathrm{~s}$ exposure time and covering the entire Lyman series plus O I, C II and S Vi (temperature range from 15000 to $200000 \mathrm{~K}$ ) has also been explored showing the response of all these lines during transient phenomena. New common features linking explosive events and blinkers were found. During explosive events, the central intensity increases between 1.6 and 2.0 times the pre-event value while the same range of intensity increase was already reported during blinker phenomena. On the other hand the maximum intensity increase in Ly 6 was only $13 \%$.
\end{abstract}

Key words. Sun: activity - Sun: atmosphere - Sun: magnetic field - Sun: UV radiation

\section{Introduction}

Observations made with the Naval Research Laboratory's high resolution telescope and spectrograph (HRTS) revealed the existence of high velocity small-scale events seen in lines from ions formed at temperatures from $2 \times 10^{4} \mathrm{~K}(\mathrm{C}$ II $)$ to $2 \times 10^{5} \mathrm{~K}(\mathrm{~N} \mathrm{v})$ with no signature in chromospheric lines such as $\mathrm{Si}$ II $\left(1.3 \times 10^{4} \mathrm{~K}\right)$, $\mathrm{C}$ I $\left(6-10 \times 10^{3} \mathrm{~K}\right)$ and $\mathrm{O}$ I $\left(1.5 \times 10^{4} \mathrm{~K}\right)$, formed below $2 \times 10^{4} \mathrm{~K}$ (Brueckner \& Bartoe 1983). Discovered and classified by Brueckner \& Bartoe (1983) as turbulent events and jets, they are characterized by non-Gaussian profiles due to an enhancement in the blue and red wings, often observed with an offset along the slit (Dere et al. 1989; Innes et al. 1997a). Most of the events are predominantly blueshifted, showing velocities up to $250 \mathrm{~km} \mathrm{~s}^{-1}$. Their first identification was followed by several studies based on observations obtained with HRTS and the spectrometer Solar Ultraviolet Measurements of Emitted Radiation (SUMER) on-board SoHO performed by Dere et al. (1989), Porter \& Dere (1991), Innes et al. (1997a), Chae et al. (1998a), Pérez et al. (1999), Landi et al. (2000) and Teriaca et al. (2001). The authors extended the description of their general characteristics naming them by the term "explosive events".

The explosive events are located in the network lanes at the boundaries of the super-granulation cells where the

Send offprint requests to: M. S. Madjarska,

e-mail: madj@star.arm.ac.uk neutral line separates regions of opposite magnetic polarity (Dere et al. 1991; Porter \& Dere 1991). They appear preferably in regions with weak fluxes of mixed polarity or on the border of regions with large concentration of magnetic flux (Chae et al. 1998a). The average lifetime of the explosive events ranges from $\sim 60$ to $350 \mathrm{~s}$ (Dere 1994 ) with spatial dimensions of $\sim 2500 \mathrm{~km}$. They are often observed in bursts (Innes et al. 1997a) lasting up to $30 \mathrm{~min}$ in regions undergoing magnetic cancellation (Chae et al. 1998a). Using the density sensitive line intensity ratio O IV 1401.16/1404.81, Teriaca et al. (2001) found an electron density increase by a factor $\sim 3$ during explosive events. They also showed an increase of the line intensity ratio O IV 1401/O III 703 during an explosive event which suggests a temperature increase during the phenomena.

Explosive events seem to be a product of magnetic reconnection (Parker 1988; Porter \& Dere 1991; Dere 1994; Innes et al. 1997b; Wilhelm et al. 1998; Roussev et al. 2001) because they tend to occur on the neutral line separating regions of opposite magnetic polarity, appear as bi-directional jets (Innes et al. 1997b) with velocities comparable to the local Alfvén velocity (Dere 1994), and they are often associated with a cancellation of photospheric magnetic flux (Dere et al. 1991; Dere 1994; Chae et al. 1998a).

After the launch of the SoHO satellite it became possible to observe Lyman lines with high spectral, spatial and temporal resolution. Using these lines as a plasma diagnostic tool has several advantages. They cover heights 
with a strong temperature gradient and therefore can be used to diagnose the time evolution of different temperature structures. The cores of these optically thick lines are supposed to be formed in the chromosphere or at the base of the transition region while the wings are formed over large atmospheric depths in regions far below the core formation i.e. in the lower chromosphere (Vernazza et al. 1981). It was demonstrated by Heinzel et al. (1997) that the cores of the higher Lyman lines are sensitive to temperature because the upper levels of the corresponding transitions are strongly coupled to the kinetic temperature while the wings show sensitivity to the gas pressure. These lines are relatively strong and thus can be used for observations which need short exposures as in the case of explosive events. However, the observed profiles can only be interpreted correctly if multilevel NLTE (Non LocalThermodynamic-Equilibrium) radiative transfer calculations are performed to explain the registered line shapes and intensities. That is why the present study is mainly based on the response of these lines during an explosive event through the emission changes in the blue and red wings of the registered lines.

Recently, Chae et al. (1998b), using SUMER data in Si IV $1402 \AA$ and Big Bear Solar Observatory (BBSO) $\mathrm{H} \alpha$ spectrograph observations, found that chromospheric upflow events rising in intranetwork areas are related to transition region explosive events. To support the idea of the chromospheric origin of some of the explosive events full spectrum observations covering lines with a wide range of formation temperature $\left(1.5 \times 10^{4}-2 \times 10^{5} \mathrm{~K}\right)$ have been analysed.

A new transient phenomenon observed with the Coronal Diagnostics Spectrometer (CDS) on-board SoHO as enhancements in the flux of transition region lines at network junctions was recently introduced by Harrison (1997) named as "blinkers". Blinkers are mainly observed in lines of $\mathrm{O}$ III, $\mathrm{O}$ IV and $\mathrm{O} \mathrm{V}$ while lines formed at lower and higher temperatures (such as He I and Mg X) show only a modest increase in intensity. They show a typical lifetime of $\sim 17$ min over an area of $\sim 5 \times 10^{7} \mathrm{~km}^{2}$, with an average intensity increase in $\mathrm{O} v$ of $\sim 1.5$ which, in extreme cases, can reach values as high as five times the pre-event level (Harrison et al. 1999). Chae et al. (2000) used CDS and SUMER coordinated observations in the transition region lines O v $629 \AA$ (CDS) and Si IV $1402 \AA$ (SUMER), and Big Bear Solar Observatory magnetograms to examine the relation between blinkers and explosive events showing similarities such as line profiles with enhanced wings, but on a different scale, suggesting the same physical origin of the two events, namely magnetic reconnection, but with different magnetic geometries.

This paper presents a study on the temporal evolution of a plasma with different temperatures during explosive events. In the next section we discuss the observations and the data reduction. Section 3 presents the data analysis, paying attention on the line blends and event identification. The results are described in Sect. 4 and the conclusions in Sect. 5.

\section{Observations and data reduction}

In search of high cadence observations obtained in transition region lines, we selected a series of temporal observations from the SoHO archive performed with the SUMER spectrometer in S VI (933.38 $\AA$ ) and Ly 6 (930.748 $\AA$ ), and a full spectrum temporal series, covering all Lyman lines from Ly 4 up to the end of the series. The SUMER instrument is a high resolution stigmatic normal incidence spectrometer covering the wavelength range from $\sim 400$ to $1610 \AA$ (Wilhelm et al. 1995, 1997; Lemaire et al. 1997) with a spatial resolution $\sim 1.5^{\prime \prime}$ and spectral pixels size between $42 \mathrm{~m} \AA$ and $45 \mathrm{~m} \AA$. The datasets have been obtained on 1996 October 17 and 18 and 1997 June 5, pointing the instrument on the "quiet Sun" at heliographic coordinates $X=0^{\prime \prime}$ and $Y=0^{\prime \prime}$ and $X=696^{\prime \prime} 2$ and $Y=158^{\prime \prime} 3$, respectively. In both cases the slit $1^{\prime \prime} \times 120^{\prime \prime}$ was used.

The observations in 1996 (DS1) started on October 17 at 19:23:11 UT and finished on October 18 at 00:23:39 UT. The spectra were obtained exposing a band of 120 spatial $\times 1024$ spectral pixels (spectral range from 907 to $954 \AA$ ) on the central part of detector B for 200 s. A temporal sequence consisting of two spectral windows (120 spatial $\times 50$ spectral pixels) centered on Ly 6 $930.748 \AA$ and S VI $933.38 \AA$ with 10 s exposure time was telemetred to the ground as well. No compensation for the solar rotation was applied and therefore for a solar rotation at this heliographic latitude of about $10^{\prime \prime} / 1 \mathrm{~h}$ the slit covered a region of $\sim 47^{\prime \prime}$.

The observations on 1997 June 5 (DS2) were obtained between 12:05:13 UT and 14:15:27 UT exposing for $30 \mathrm{~s}$ 120 spatial $\times 1024$ spectral pixels on detector B covering the wavelength range from 903 to $943 \AA$. This wavelength range includes the Lyman lines from Ly 5 up to the series limit, N IV, S VI and a few O I lines. In order to remain on the same position on the Sun a rotation compensation has been applied setting the tracking system to offset for the $X$-pointing by $0.75^{\prime \prime}$.

The reduction of SUMER raw images followed several stages such as local gain correction, flat-field subtraction and a correction for geometrical distortion. The signal to noise level is determined by the photon statistics.

Michelson Doppler Imager (MDI) (Scherrer et al. 1995) observations have been performed on 1996 October 17 and 18. The high resolution photospheric longitudinal magnetograms $\left(0.6^{\prime \prime}\right.$ per pixel) were obtained with $60 \mathrm{~s}$ exposure time and field of view of $500 \times 1024$ pixels covering all the SUMER observing period. Magnetic cancellation during explosive events cannot be registered because of the high noise level (20 Gauss rms) of the MDI. Nevertheless these observations give a general picture of explosive events formation with respect to the solar magnetic network lanes and larger concentration of magnetic flux (Fig. 1, right panel). 

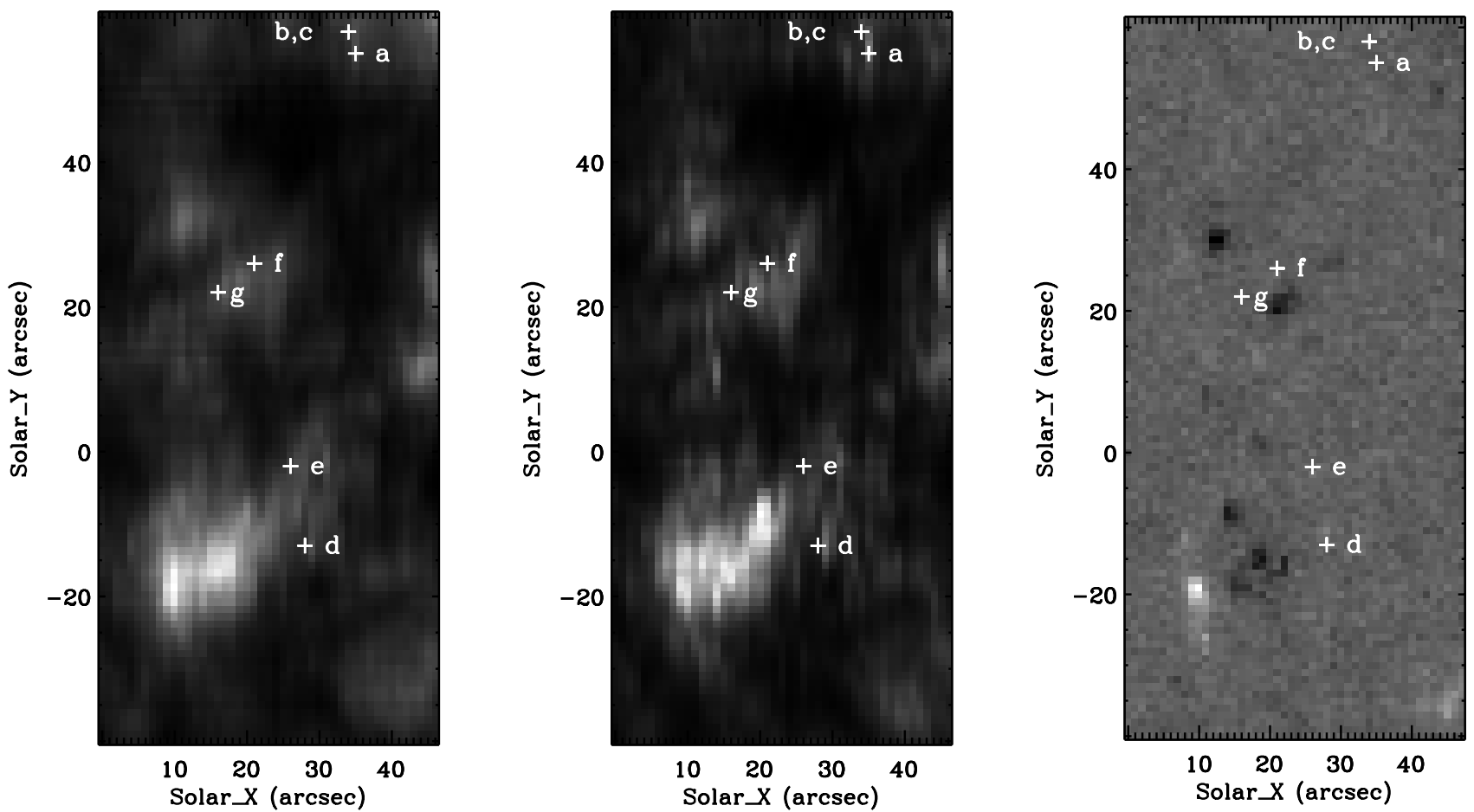

Fig. 1. Ly 6 (left) and S VI (middle) integrated line intensity images showing the network pattern and the locations of the explosive events marked by crosses and letters together with a MDI high resolution magnetogram (right) showing the position of explosive events with respect to magnetic flux concentrations. Note that the events "b" and "c" appear on the same position on the raster because we integrate over $390 \mathrm{~s}$ to reproduce each position of the slit on the Sun.

\section{Data analysis}

DS1 consists of a full spectrum obtained with $200 \mathrm{~s}$ integration time, prior to every 121 temporal spectra, covering the wavelength range from 907 to $954 \AA$. The spectra cover a few unblended O I lines such as O I $929.52 \AA$ and O I $936.63 \AA$ which have been used as a wavelength standard during the calibration. Since the reference lines are in general rather weak, we used profiles averaged along the slit to determine the centroids of these lines from a Gaussian fit. Both Ly 6 and S vi lines were registered during DS1 on the KBr part of the detector, and despite the short exposure time the signal-to-noise ratio is good. During DS2, however, the S VI line has been registered on the bare part, but the longer exposure still results in good photon statistics.

\subsection{Line blends}

Our study was based on the spectral lines listed in Table 1. In the present paper the Lyman lines (H I Ly \#) will be written as Ly \# for convenience, where \# is the serial number of the line. Unfortunately, the lines belonging to the registered wavelength range are not free of contribution from other emission lines, mainly He II $(T=5.0 \times$ $\left.10^{4} \mathrm{~K}\right)$ and/or $\mathrm{O}$ I $\left(T=1.5 \times 10^{4} \mathrm{~K}\right)$ lines. Table 1 gives the blends as identified by Curdt et al. $(1997,2001)$ and Warren et al. (1998). The Ly 8 line is excluded from our analysis because it is blended by N IV lines which have a
Table 1. The analysed lines and their corresponding blends.

\begin{tabular}{lll}
\hline Lines & $\lambda(\AA)$ & \multicolumn{1}{c}{ Blend } \\
\hline Ly 11 & 918.129 & He II 917.74 \\
Ly 10 & 919.35 & O I 919.65 \\
Ly 9 & 920.963 & He II 920.62 \\
Ly 7 & 926.84 & $\begin{array}{l}\text { He II } 925.84 \\
\text { O I } 926.295\end{array}$ \\
& & O I 930.26 \\
Ly 6 & 930.748 & He II 930.32 \\
& & O I 930.886 \\
& & Ne VII 465.22 \\
S VI & 933.378 & He II 933.44 \\
& & - \\
O I & 929.52 & - \\
O I & 936.63 & - \\
C I & 903.63 & - \\
C I & 903.96 & - \\
C I & 904.14 & - \\
C I & 904.48 & \\
\hline
\end{tabular}

strong contribution in the very bright network and especially during explosive events.

The Ly 6 line is not effected by Ne VII $465.22 \AA$ appearing in second order since the sensitivity of SUMER below $500 \AA$ is very low. It is, however, blended by a first order O I $930.886 \AA$ in the red wing, which could not be resolved. In the blue wing of Ly 6 are also O I $930.26 \AA$ and He II $930.32 \AA$ lines which appear as one feature and could be subtracted. These lines have a small, insignificant contribution of less than $10 \%$ to the blue wing of 
Ly 6 (Marsh et al. 1999). Our analysis is performed in a way (described in Sect. 3.2) ensuring that these blends do not influence the resulting response of the wings during explosive events. The other Lyman lines have small blends which do not effect our analysis and subsequent conclusions.

The $\mathrm{S}$ VI line at $933.38 \AA$ is blended in the red wing by He II Balmer 12 at 933.44 (Curdt et al. 1997, 2001). The blend effect is variable, ranging between 10 and $20 \%$ (W. Curdt, private communication). It can only be responsible for an overestimation of the count rate in the red wing of S VI. On the blue wing at $932.9 \AA$ there is a faint unidentified line (W. Curdt, private communication) which has insignificant contribution to S VI. Both O I lines as well as the $\mathrm{C}$ II lines are clean from any blends.

\subsection{Event identification}

Whereas CDS blinkers have already been associated with small-scale short-lived SUMER "unit brightening events" with a size of a few arc seconds and a lifetime of a few minutes (Chae et al. 2000; Teriaca et al. 2001) characterised by profiles with enhanced wings, explosive events show broader short- and longward shifted wings with velocities higher than $50 \mathrm{~km} \mathrm{~s}^{-1}$. As will be shown later in this paper, the central line intensities, however, show a strong intensity increase in explosive events just like in blinkers, which has been reported to increase by about a factor of two, depending on the emission line under consideration (Harrison et al. 1999). From the point of view of spatial relationship, blinkers are found in the center of the network junctions (Harrison et al. 1997, 1999; Chae et al. 2000; Teriaca et al. 2001) while the explosive events tend to appear on the border of the network junctions and the super-granulation cells. Besides the location, the two events have also different size and duration. That is why a precise identification through a visual inspection is needed to correctly select each single event.

We selected a few explosive events by visual inspection of both S VI and Ly 6 slit images in the DS1, using the above mentioned wings at velocities $>50 \mathrm{~km} \mathrm{~s}^{-1}$ as a criterion.

As was mentioned above, the observations were performed without applying rotational compensation, so the slit pointing at $X=0^{\prime \prime}$ and $Y=0^{\prime \prime}$ during $5.05 \mathrm{hr}$ of observations resulted in a drift raster scan with a width of about $47^{\prime \prime}$. Figure 1 shows the reproduced rasters in both Ly 6 and $\mathrm{S}$ VI lines with the corresponding locations of the explosive events marked by crosses and letters. The network pattern is visible on both rasters, less clear in Ly 6 due to the optical thickness of the Lyman line. An MDI image is also presented on this figure showing the region covered by the SUMER slit.

The events have a dimension along the slit Solar_ $Y \sim$ $4^{\prime \prime}-5^{\prime \prime}$ appearing in the network lanes away from larger concentration of magnetic flux. At the observed heliographic latitude the $1^{\prime \prime}$ slit needs about $390 \mathrm{~s}$ to move to
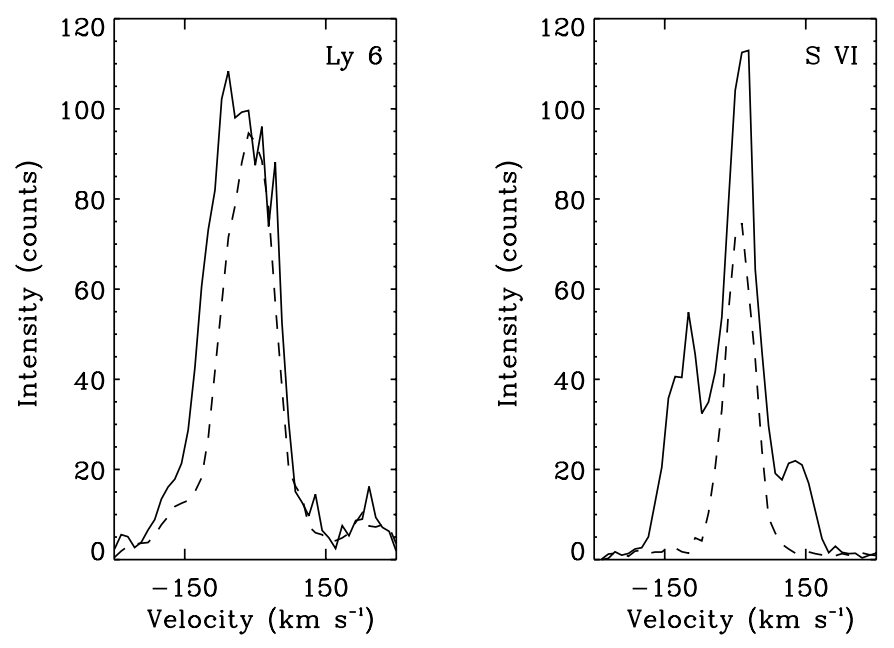

Fig. 2. Ly 6 and S VI profiles in explosive event "a" during (solid line) and before the event (dashed line), obtained by binning over 7 pixels along the slit.

the neighboring position on the Sun which makes it impossible to evaluate the Solar_ $X$ dimension of the events.

In order to follow the temporal evolution of the phenomena through the emission changes in both wings, the total emission in the wings was estimated as the line intensity over $0.17 \AA$ wide intervals centered $0.22 \AA$ away from the central position on the blue and red sides of the spectral line. In this way we avoid most of the contribution of the blends. In the case of the Ly 6 line, the blends He II $930.32 \AA$ and O I $930.26 \AA$ are observed as one feature at $0.44 \AA$ from the center of Ly 6 . This blend was evaluated to be only $\sim 3 \%$. In order to evaluate the central intensity increase, the centroids of the lines have been determined by a Gaussian fit of the line profile averaged along the slit in absence of high velocity events. The central intensity is that obtained within $0.13 \AA$ around the central position.

After the visual identification, the duration of a single explosive event was defined as the period of a strong increase of the integrated emission in one of the wings until its decay.

\section{Results}

The DS1 consists only of one Lyman line (Ly 6) and the $\mathrm{S}$ VI line, but this temporal series has the advantage of a high temporal resolution (10 s exposure time) and containing two lines which have one order of magnitude difference in formation temperature. These two facts permit us to determine whether Ly 6 shows a response to explosive events, and if it does, what is the line profile shape and what is the temporal evolution of a different temperature plasma during these transient events?

First the strongest event was analysed, because a response of the optically thick Lyman lines could only be identified when the event is so strong that its emission is shifted far enough to be visible against the high background emission (Brueckner \& Bartoe 1983). Figure 2 

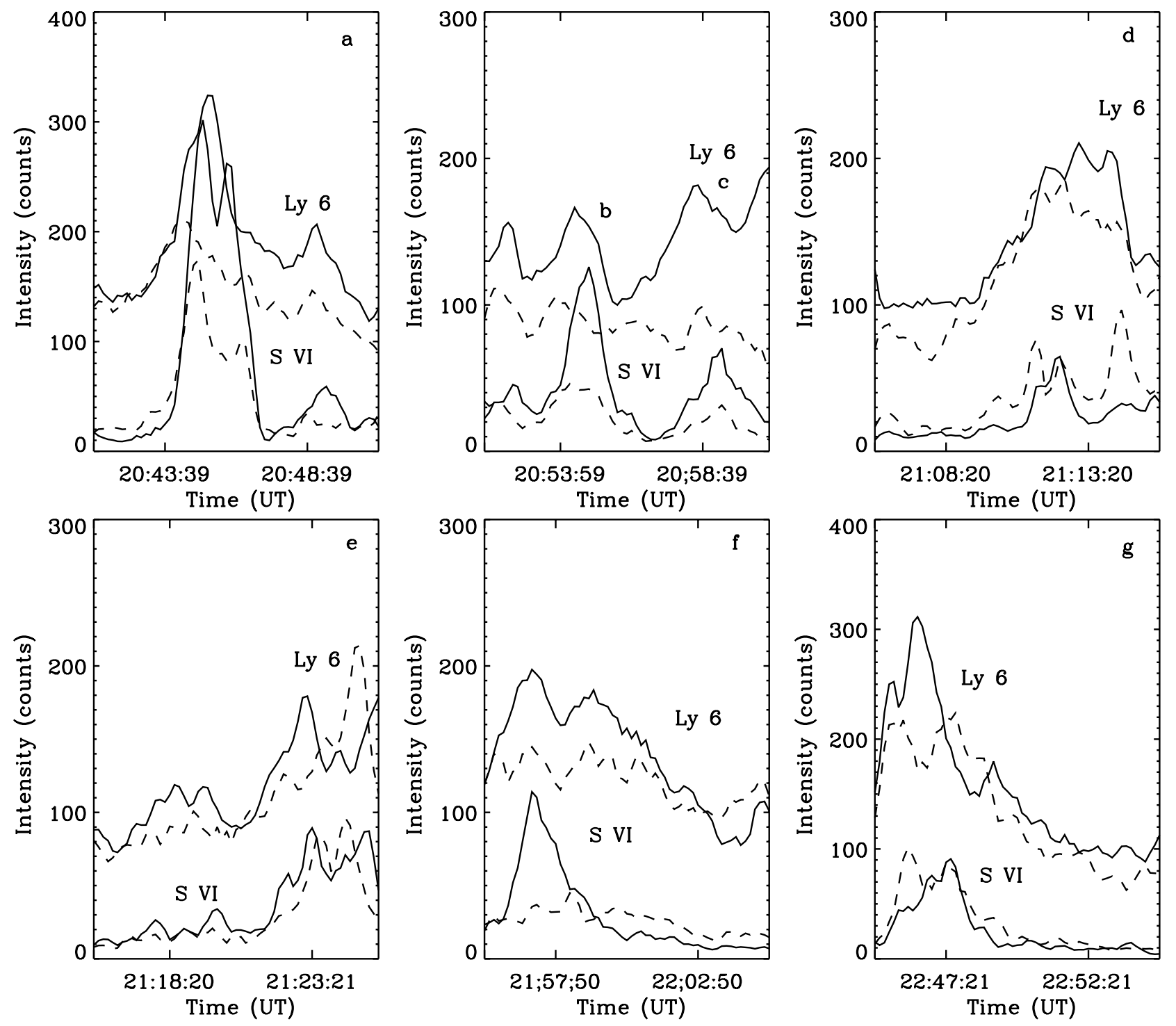

Fig. 3. Integrated intensity in the blue (solid line) and red (dashed line) wings of Ly 6 and S vi during the explosive events. The beginning of the events " $\mathrm{f}$ " and "g" are not registered because a reference spectrum was obtained before each temporal series.

shows Ly 6 and S VI profiles during the event. We overplotted a pre-event profile in order to visualise the line shape and intensity changes. An increase in the blue wing in Ly 6 is clearly visible whereas the central intensity of the line does not show any significant change. The analysis of all line profiles showed that an increase in Ly 6 only becomes visible when at the same time the S vi central intensity increases by $\sim 100 \%$ or more above the pre-event value.

All identified events were searched for a Lyman line response through the emission increase in the blue and red wings. In order to identify the start of a single event the background emission in the wing before or/and after the event was evaluated first. Note that the growth of the intensity in the wings for the two lines is different because of the different optical thickness of the Ly 6 and $\mathrm{S}$ vi lines. Through a visual inspection we determined the beginning of each event. It was defined as a point from the ascending branch of the intensity curve showing an obvious increase above the background emission. In order to improve the significance of the time delay measurements a few points above the starting one were also evaluated. Figure 3 presents the smoothed integrated intensity curves for all the registered events. The time differences for all events are given in Table 2. The time difference of the intensity peaks are easy distinguishable for events "a", "b", "c", and "e". The blue wing of event "d" has a very complex behaviour and it is difficult to define its starting time. Events "f" and "g" already started before the observations begun (remember that a reference spectrum was obtained before each 121st spectrum). The time differences for these events are also given in Table 2, but have to be considered as lower limits. The general picture of the evolution of all events is characterised by a blueshift accompanied by a redshift with the emission in the red wing often lower than in the blue one. 
Table 2. Time difference in the response of the blue and red wing of Ly 6 compared to the $\mathrm{S}$ vi line.

\begin{tabular}{ccc}
\hline Event & $\begin{array}{c}\text { Blue wing } \\
\text { Time }(\mathrm{s})\end{array}$ & $\begin{array}{c}\text { Red wing } \\
\text { Time }(\mathrm{s})\end{array}$ \\
\hline $\mathrm{a}$ & $40 \pm 9$ & $40 \pm 2$ \\
$\mathrm{~b}$ & $13 \pm 6$ & $11 \pm 1$ \\
$\mathrm{c}$ & $79 \pm 21$ & $33 \pm 10$ \\
$\mathrm{~d}$ & - & $19 \pm 6$ \\
$\mathrm{e}$ & $31 \pm 14$ & $16 \pm 4$ \\
$\mathrm{f}$ & $39 \pm 7$ & - \\
$\mathrm{g}$ & $16 \pm 4$ & $28 \pm 6$ \\
\hline
\end{tabular}

Concerning the intensity changes of the stationary component of the line we have determined a mean intensity increase in S VI of about 0.6 above the pre-event intensity. During the strongest one it reaches 0.9-1.0. However, only very small changes of the order of $\sim 0.13$ have been registered in Ly 6 due to the optical thickness of this line. Therefore, the observed self-absorption during the explosive events in Lyman lines may be mainly due to an emission increase in the wings. Figure 4 shows the intensity changes of the stationary component obtained in the way as described in Sect. 3.2. As was mentioned already, Harrison et al. (1999) have registered the same intensity increase of $0.2-0.3$ above the pre-event value in the optically thick He I $584 \AA$ and 1.0 in optically thin lines such as O v $629 \AA$, O III $599 \AA$ and O IV $554 \AA$ during blinker transient phenomena.

We defined the duration of a single event as the time from the intensity increase in the wing until its decay. Defined in that way, it was found that events with duration of more than $100 \mathrm{~s}$ show spikes in the wing intensity curve as was already observed by Dere et al. (1991). They are found to last between 40 and $100 \mathrm{~s}$. Similar peaks, but on a different time scale, have also been observed during blinker events (Harrison et al. 1999). The authors suggested that this could be the result of the superimposed intrinsic solar variability.

The strong response of Ly 6 rises the question about the registration of the explosive events at lower temperature (below $2 \times 10^{4} \mathrm{~K}$ ). To explore this idea we searched a full spectrum temporal series covering simultaneously Lyman lines $(<20000 \mathrm{~K})$, O I $(\sim 15000 \mathrm{~K})$ lines and S VI $(\sim 200000 \mathrm{~K})$ line for explosive events. Only one event has been selected. Figure 5 displays the line profiles of the Ly 11 and S VI lines together with the corresponding slit negative images. The profiles were obtained by binning over 3 spectra and 5 pixels along the slit (note that S VI is registered on the bare part of detector B). The line profile before the explosive event does not show the presence of the faint feature at $932.9 \AA$ and therefore we do not expect any significant contribution during the explosive event. Some of the Lyman lines show strong selfabsorption, but as was mentioned above, this may be due to an increase of the emission in the wings as the central intensity slightly increases during the event.
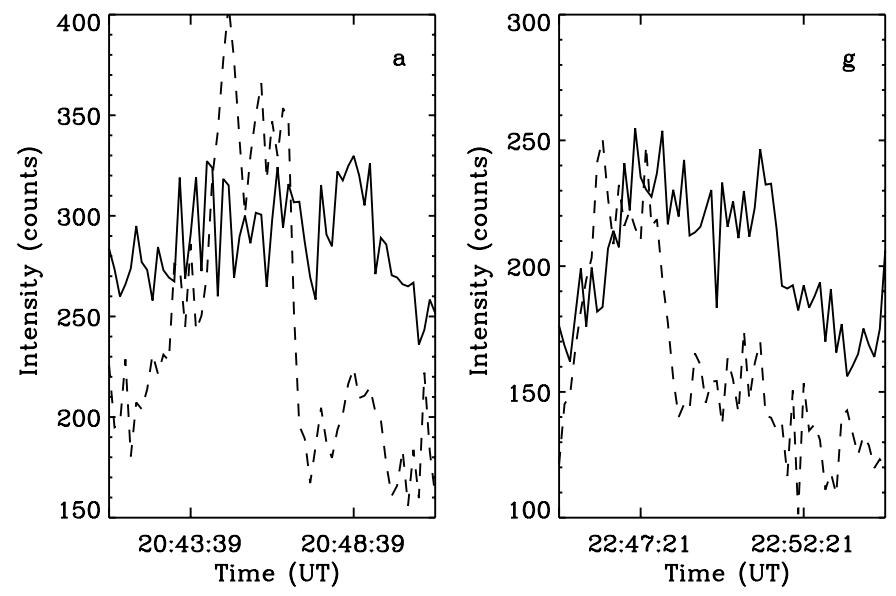

Fig. 4. Two examples of the stationary component intensity changes of the Ly 6 (solid line) and S vi (dashed line) lines during explosive events "a" and "g".

Figure 6 shows the integrated intensity in the blue wings of Ly 11, Ly 10, Ly 9, Ly 7, Ly 6 and S VI obtained as described in Sect. 3.2. The total intensity of $\mathrm{C}$ II includes the intensity of four blended C II lines at 903.59, 903.99, 904.14, 904.46 ̊ (Curdt et al. 1997, 2001). We were especially interested in the response of the $\mathrm{O}$ I $(15000 \mathrm{~K})$ lines. The visual inspection of these lines suggested some intensity increase during the explosive event, but this is uncertain because of the low emission of these lines. Therefore we used the total intensity in the two selected unblended oxygen lines O I 929.52 and $936.63 \AA$. As was shown above, the central intensity of optically thin lines increases by at least 1.6 and in optically thick lines by less than 1.2 times during an explosive event. Therefore, when it is impossible to detect blueshifted and redshifted emission as in the case of faint lines such as oxygen, and the event is already registered by other simultaneously recorded lines, the total intensity in these lines is a good indicator for the presence of the event in this spectral line. As can be seen from Fig. 5, the continuum also shows an increase during the explosive event, which made it necessary to evaluate and subtract the background for each line. This is especially important in the case of the faint oxygen lines. The plots in Fig. 6 show that during the event, a plasma with a temperature from 15000 to $200000 \mathrm{~K}$ is registered. Unfortunately, the longer exposure time during these observations does not permit us to follow the temporal evolution of the different temperature plasma.

\section{Conclusions}

During the last decades numerous solar activity phenomena (solar flares, surges, sprays etc.) have been considered as manifestation of magnetic reconnection processes (Giovanelli 1946; Gold \& Hoyle 1960; Priest 1981; Parker 1988; Forbes 1991; Priest \& Forbes 2000) resulting from the formation of a current sheet formed by magnetic flux 

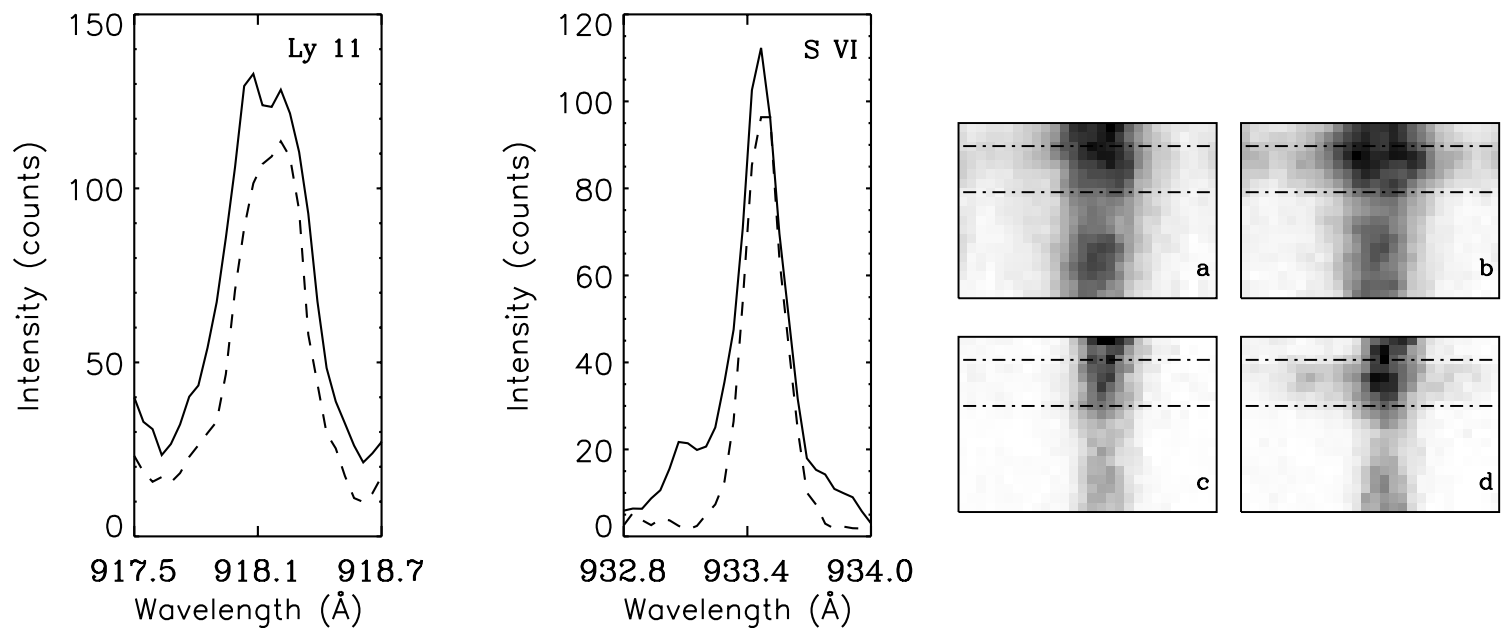

Fig. 5. Ly 11 and S VI line profiles before (dashed line) and during (solid line) the explosive event. The profiles are obtained by binning over 3 spectra and over the region along the slit as shown by the horizontal lines on the right panel slit negative images. Right panels: slit negative images before and during the explosive event in Ly $11(\mathbf{a}), \mathbf{b})$ ) and S VI (c), d)), respectively.
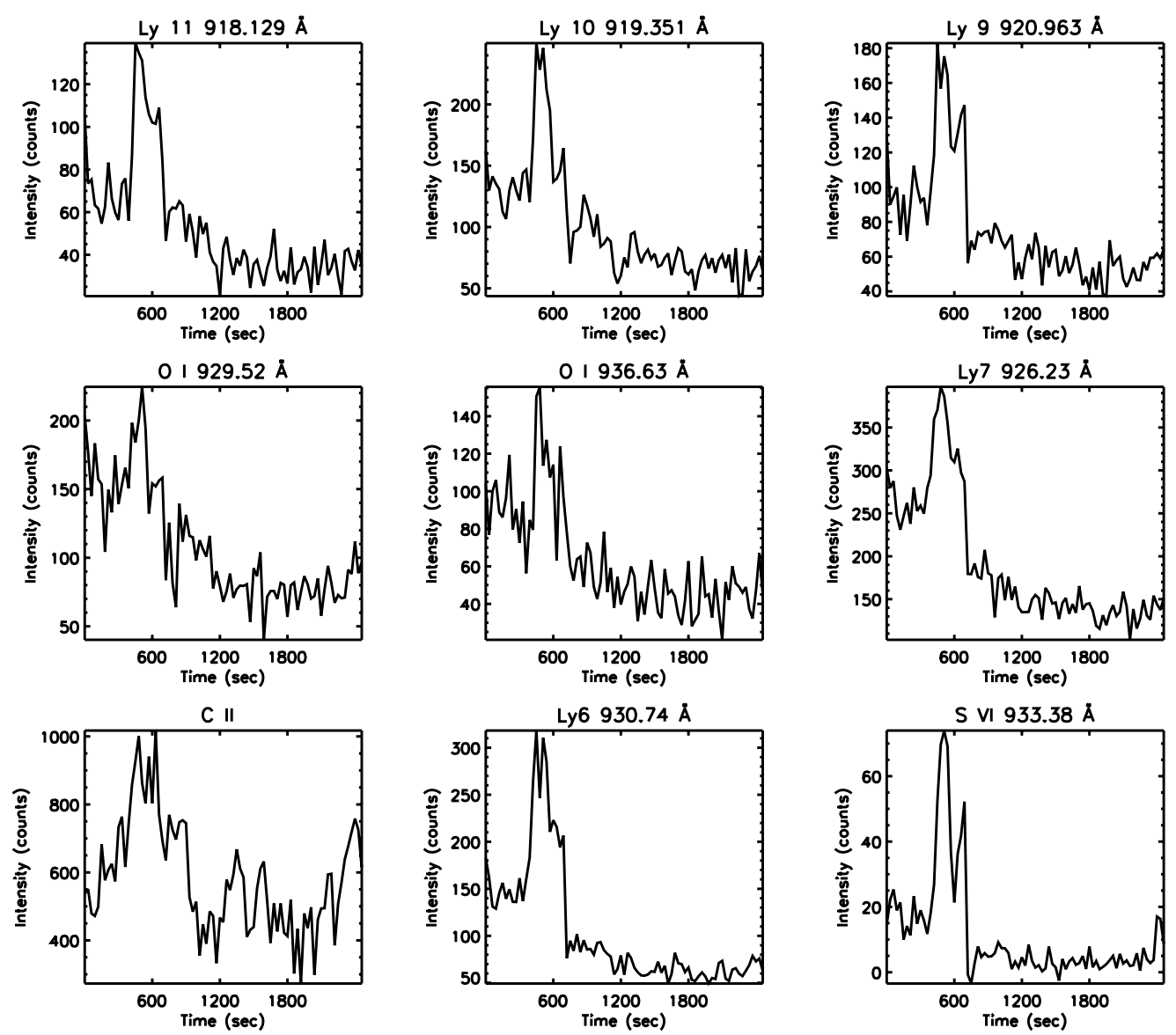

Fig. 6. Integrated intensity in the blue wing of Ly 11, Ly 10, Ly 9, Ly 7, Ly 6 and S VI in the region of the explosive event ( 5 pixels along the slit) for the all analysed dataset. We also show the total intensity of the $\mathrm{O}$ I and $\mathrm{C}$ II (includes four blended C II lines) lines.

tubes with opposite polarities pushed together by intensive photospheric motions. Such a current sheet may also occur between a new emerging flux tube and a pre-existing one. It is believed that the explosive events appearing as bi-directional jets with velocities comparable to the local
Alfvén velocity are such events resulting from magnetic reconnection. This belief is due to their appearance on the super-granulation cell boundaries and the fact that they are often associated with the cancellation of the photospheric magnetic field. 
Every explosive event shows some particularities and it is not possible to define a general picture of their behaviour. Two datasets complementing each other have been explored in this study trying to identify new common features for all of the events. We have examined the temporal evolution of different temperature plasmas during explosive events taking the advantage of having registered these phenomena with a short exposure time (10 s) in lines with one magnitude difference in the formation temperature. Another temporal series but covering simultaneously lines with a wide range of formation temperatures has been used to find out which temperature plasma is involved in this phenomenon. Our analysis revealed that the explosive events appear first at chromospheric temperatures. After some time delay which differs from event to event a hotter plasma is registered.

Solar flares are another solar activity phenomena, resulting from magnetic reconnection. During their impulsive phase they are also registered in many EUV spectral lines ranging from neutral hydrogen and oxygen to highly ionised ions of metals (Priest 1981 and the citations therein). Results, with quite large uncertainties, from OSO-III observations (Hall 1971) suggest that probably similar time delays in the response of EUV emission lines exist during solar flares as well. The similarities between explosive events and flares suggest a common mechanism of their generation. This may help to find out why a plasma exists at such a wide temperature range during explosive events although not necessarily at coronal temperatures and how that is related to their generation.

Our study has been based on only a few events. In order to define the time evolution of plasma with different temperatures during explosive events further spectroscopic observations simultaneously covering lines with a wide range of formation temperatures obtained with high temporal resolution (exposure time around $10 \mathrm{~s}$ ) are needed. Koutchmy et al. (1997) provided evidence for the existence of a new type of soft X-ray brightening event with duration and size comparable to the explosive events and called them "soft X-ray coronal flashes". To find out whether these phenomena are the coronal counterpart of the explosive events simultaneous multi-instrumental and spectroscopic multi-wavelength observations have to be obtained.

Such observations are also needed to explore the connection and similarities between explosive events and blinkers. Chae et al. (1998) using SUMER and CDS observations have already revealed some similarities between explosive events and blinkers. CDS blinkers have been associated with small-scale short-lived SUMER "unit brightening events" with a size of a few arcsec and a lifetime of a few minutes (Chae et al. 2000; Teriaca et al. 2001) characterised by profiles that are not as broad as those of explosive events but still with significantly enhanced wings. In this work two more common features of the two events have been identified. We compared the intensity increase of the "line at rest" during explosive events and blinkers (reported by Harrison et al. 1999) and found out that the same intensity increase takes place during both events. The existence of intensity peaks during an explosive event, registered also with HRTS by Dere et al. (1989), is another link between explosive events and blinkers.

Further efforts are required regarding time delays of the response of different spectral lines during explosive events as well as a more detailed NLTE study concerning the line formation of optically thick lines such as the Lyman series. The present observational dataset suggests a time delay with the chromospheric feature being detected first as a blueshifted plasma several seconds before a response to the magnetic reconnection is observed at transition region temperatures.

Acknowledgements. Research at Armagh Observatory is grantaided by the N. Ireland Dept. of Culture, Arts and Leisure, while partial support for software and hardware is provided by the STARLINK Project which is funded by the UK PPARC. This work was supported by PPARC grant PPA/GIS/1999/00055. The SUMER project is financially supported by DLR, CNES, NASA, and PRODEX. Thanks to Prof. E. Priest and Dr. W. Curdt for fruitful discussions.

\section{References}

Brueckner, G. E., \& Bartoe, J.-D. F. 1983, ApJ, 272, 329

Chae, J., Wang, H., Lee, C. Y., Goode, P. R., \& Schühle, U. 1998a, ApJ, 497, L109

Chae, J., Wang, H., Lee, C. Y., Goode, P. R., \& Schühle, U. 1998b, ApJ, 504, L123

Curdt, W., Feldman, U., Laming, J. M., et al. 1997, A\&AS, 126,281

Curdt, W., Brekke, P., Feldman, U., et al. 2001, A\&A, 375, 591

Dere, K. P., Bartoe, J.-D. F., \& Brueckner, G. E. 1989, Sol. Phys., 123, 41

Dere, K. P., Bartoe, J.-D., Brueckner, G. E., Ewing, J., \& Lund, P. 1991, J. Geophys. Res., 96, 9399

Dere, K. P. 1994, Adv. Space Res., 14(4), 13

Forbes, T. G. 1991, Geophys. Astrophys. Fluid Dyn., 62, 15

Giovanelli, R. G. 1946, Nature, 158, 81

Gold, T., \& Hoyle, F. 1960, MNRAS, 120, 89

Hall, L. A. 1972, Solar Phys., 21, 1971

Harrison, R. A., Sawyer, E. C., Carter, A. M., et al. 1995, Sol. Phys., 162, 233

Harrison, R. A. 1997, Sol. Phys., 175, 467

Harrison, R. A., Fludra, A., Pike, C. D., et al. 1997, Sol. Phys., 170,123

Harrison, R. A., Lang, J., Brooks, D. H., \& Innes, D. E. 1999, A\&A, 351, 1115

Heinzel, P., Schmieder, B., \& Vial, J.-C. 1997, Proc. Fifth SoHO Workshop (ESA SP-404); Nordwijk: ESA, 427

Innes, D. E., Brekke, P., Germerott, D., \& Wilhelm, K. 1997a, Sol. Phys., 175, 341

Innes, D. E., Inhester, B., Axford, W. I., \& Wilhelm, K. 1997b, Nature, 386, 811

Koutchmy, S., Harra, H., Suematsu, Y., \& Reardon, K. 1997, A\&A, 320, 33

Lemaire, P., Wilhelm, K., Curdt, W., et al. 1997, Sol. Phys., 170, 105

Marsh, E., Tu, C.-Y., Heinzel, P., Wilhelm, K., \& Curdt, W. 1999, 347, 676

Parker, E. N. 1988, ApJ, 330, 474 
Pérez, M. E., Doyle, J. G., Erdèlyi, R., \& Sarro, L. M. 1999a, A\&A, 342, 279

Porter, J. G., \& Dere, K. P. 1991, ApJ, 370, 775

Priest, E. R. 1981, Solar Flare Magnetohydrodynamics (Gordon \& Breach, London)

Priest, E. R., \& Forbes, T. 2000, Magnetic Reconnection: MHD theory and applications (Cambridge Univ. Press )

Roussev, I., Galsgaard, K., Erdélyi, R., \& Doyle, J. G. 2000, A\&A, 370, 298

Scherrer, P. H., Bogart, R. S., Bush, R. I., et al. 1995, Sol. Phys., 162, 129

Teriaca, L., Madjarska, M. S., \& Doyle, J. G. 2001, Sol. Phys., 200, 91
Vernazza, J. E., Avrett, E. H., \& Loeser, R. 1981, ApJS, 45, 635

Warren, H. P., Mariska, J. T., \& Wilhelm, K. 1998, ApJS, 119, 105

Wilhelm, K., Curdt, W., Marsch, E., et al. 1995, Sol. Phys., 162,189

Wilhelm, K., Lemaire, P., Curdt, W., et al. 1997, Sol. Phys., 170, 75

Wilhelm, K., Innes, D. E., Curdt, W., Kliem, B., \& Brekke, P. 1998, in Solar Jets and Coronal Plumes, Guadalupe, France, ESA-SP 421, 103 